\section{A comparative study of postoperative intraocular pressure changes in small incision vs conventional extracapsular cataract surgery}

PD Sharma and MR Madhavi

Department of

Ophthalmology, Mamata Medical College Khammam, Andhra Pradesh, India

Correspondence:

PD Sharma,

Department of

Ophthalmology,

Mamata Medical College,

Khammam,

Andhra Pradesh,

Pin 507002,

India

Tel: + 91983706 8919;

Fax + 915912360077 .

E-mail: drpdsharma@

yahoo.co.in

Received: 28 February 2009 Accepted in revised form: 24 June 2009

Published online: 24 July 2009

Conflict of interest: None Propritary interest: None
Abstract

Aims To compare short- and long-term IOP changes in small incision cataract surgery (SICS) vs conventional extracapsular cataract surgery (CECS).

Methods A total of 232 eyes of as many patients undergoing cataract surgery were randomized to SICS or CECS. Sixteen eyes had to be excluded out of the study. IOP was recorded preoperatively and postoperatively on day 1,2, and 7; and then after 1,3, and 6 months. Only 160 eyes remained under 6-month follow-up, out of which 48 had CECS and remaining 112, SICS. Results were analysed statistically (repeated measure ANOVA, multiple comparisons).

Results A significantly higher IOP was observed on day 1, 2, and 7 in both types of surgeries as compared to mean preoperative IOP. Variation in rise was more pronounced in CECS (nearly twofold) than in SICS $(P<0.05)$. Maximum decrease in IOP occurred during the first week and it was more rapid in SICS than CECS. At 3 months, IOP was significantly higher than baseline in CECS but significantly lower in SICS. From 3 to 6 months, there was no further decrease in IOP in either type of surgery.

Conclusions IOP rises significantly on day one in CECS and SICS and thereafter comes down slightly by day 2 and rapidly by day 7 . IOP rise is more pronounced in CECS than in SICS. After 1 week to 3 months, IOP decline is very gradual and thereafter ceases to decrease. Eye (2010) 24, 608-612; doi:10.1038/eye.2009.191; published online 24 July 2009
Keywords: intraocular pressure; conventional extracapsular cataract surgery; small incision cataract surgery; developing countries

\section{Introduction}

The scleral tunnel incision was introduced in the early 1980s in an attempt to provide better healing with less surgically induced astigmatism. This led to development of modern manual small incision sutureless cataract surgery by Blumenthal ${ }^{1,2}$ and is now widely practiced because it leads to early rehabilitation, has less learning curve in comparison to phacoemulsification technique, and is cost effective. Therefore, this method of cataract surgery has become very popular in developing countries such as India.

There are numerous reports on effect of conventional cataract surgery on intraocular pressure $^{3-12}$ but very few on small incision cataract surgery. ${ }^{13}$ Initial rise of IOP in early postoperative period after cataract surgery is well documented. ${ }^{5,8,11}$ The relatively high incidence of IOP rise occurring in the immediate postoperative period is clinically important for outcome of vision, especially in glaucoma patients as damage from transient increase of IOP could be devastating to visual outcome and reduce the benefit of cataract extraction. ${ }^{11}$

This prospective study was carried out to ascertain short and long-term IOP changes in the patients undergoing sutureless small tunnel incision cataract surgery (SICS) in comparison to conventional extracapsular cataract surgery with sutures (CECS). 


\section{Materials and methods}

In all, 232 eyes of as many individuals in the age group of 30-90 years visiting this hospital for undergoing cataract surgery were recruited for the study after obtaining approval of the ethics committee.

Cases with clear cornea and preoperative IOP $\leqslant 21 \mathrm{~mm} \mathrm{Hg}$ were only included. The exclusion criteria were preexisting traumatic, infective, or inflammatory ocular conditions, pseudoexfoliation, high refractive errors, shallow anterior chamber, congenital juvenile or complicated cataract, and scleral thinning. Other exclusion criteria were improper incision or tunnel construction, inadequate pupillary dilatation, posterior capsular rupture, vitreous loss, hyphoema, wound leak, iris prolapse, and corneal oedema.

Intraocular pressure was measured with Goldmann applanation tonometer by recording at least three readings and averaging them. All IOP measurements were carried out between 10-12 O'clock to minimize the effect of diurenal variations. Pupils were dilated with phenylephrine $5 \%$ and tropicamide $1 \%$ topical drops administered 2-3 times every $10 \mathrm{~min}$ along with flurbiprofen $0.03 \%$ drops to prevent intraoperative miosis.

All 232 surgeries were performed by three surgeons (P.D. Sharma, I.S. Murthy, P. Pradeep) who were equally adept in either technique. The cases were assigned at random to conventional or small incision cataract surgery without the knowledge of the operating surgeon. If required, incision in SICS was extended in dens hard nuclear cataracts to avoid excessive manipulation. All patients were operated under peribulbar anaesthesia.

CECS consisted of fornix-based conjunctival flap, a three-step posterior limbal 11-12 mm long three-step groove incision, anterior can opener capsulectomy or capsulorrhexis after filling anterior chamber with $2 \%$ methyl cellulose, hydrodissection, nucleus rotation and delivery, cortical aspiration, posterior chamber intraocular lens implantation in a cushion of methyl cellulose, irrigation-aspiration of the viscoelastic material, and closure of section with 4-5 interrupted $10^{\circ}$ monofilament nylon sutures.

In SICS, a standard 5.5-6.5 mm sclerocorneal tunnel flap valve incision $1.0 \mathrm{~mm}$ into clear cornea was made $1.5-2.0 \mathrm{~mm}$ behind $12^{\prime} \mathrm{O}$ clock limbus. Internal incision was about $20 \%$ larger than external incision. After anterior can opener capsulectomy or capsulorhexis, hydrodissection, and nucleus rotation, nucleus was dialled into AC and removed by methyl cellulose expression or by sandwiching between a wire vectis and a lens dialler. Cortical washout and intraocular lens implantation were done as in CECS. No sutures were applied to close the section.
All eyes received a subconjunctival injection of gentamycin and dexamethasone $(0.25 \mathrm{ml}$ each $)$ at the end of surgery. Oral antibiotics and analgesics were administered postoperatively but IOP lowering agents were avoided.

On the first postoperative day, patient was assessed for the presence of any postoperative exclusion criteria. The IOP was recorded and even if higher, no immediate IOPlowering therapy was administered. Patient was followed up on day 2 , day 7 , and subsequently at the end of 1,3 , and 6 months of surgery.

The data was analysed statistically on SPSS software (repeated measure ANOVA, F-test.) Difference between IOP values in relation to preoperative IOP within and among the categories was tested by multiple comparisons (Dunnett's and Tukey's HSD tests).

\section{Results}

Of the 232 individuals, 16 eyes were excluded under exclusion criteria and 56 dropped out of follow-up in the 6-month period. Finally 160 eyes of as many individuals were analysed. Mean age of the study sample was 62.22 years, $38.75 \%$ were men and $61.25 \%$, women (Table 1); 48 eyes had CECS and 112 had SICS. There was a steep rise in mean postoperative IOP on day 1 in both types of surgery, which persisted till day 2 , and decreased rapidly thereafter till day 7. IOP decreased gradually from 1 week till 3 months, after which it stabilized till the follow-up period of 6 months. IOP rise was more pronounced in CECS in comparison to SICS (Table 2). There were no outliers in the study.

\section{Discussion}

Although phacoemulsification with endocapsular IOL implantation is the preferred modern technique and is increasingly available in developing countries including India, it is out of reach for vast majority of the poor population dependent mainly on facilities provided by

Table 1 Age group and sex-wise distribution of patients

\begin{tabular}{lccc}
\hline Age & Male & Female & Total \\
\hline $30-39$ & 0 & 1 & 1 \\
$40-49$ & 6 & 7 & 13 \\
$50-59$ & 6 & 27 & 33 \\
$60-69$ & 25 & 30 & 55 \\
$70-79$ & 23 & 21 & 44 \\
$80-89$ & 6 & 8 & 14 \\
Total & $66(41.28 \%)$ & $94(58.75 \%)$ & 160 \\
Right eyes & & & $83(51.9 \%)$ \\
Left eyes & & & $98(48.1 \%)$ \\
\hline
\end{tabular}


Table 2 Preoperative and postoperative intraocular pressure distribution in 160 patients

\begin{tabular}{|c|c|c|c|c|c|c|c|c|c|c|}
\hline \multirow[t]{3}{*}{$I O P$} & \multicolumn{10}{|c|}{ Type of surgery (no. of eyes) } \\
\hline & \multicolumn{5}{|c|}{ CECS $(\mathrm{n}=112)$} & \multicolumn{5}{|c|}{$\operatorname{SICS}(\mathrm{n}=48)$} \\
\hline & Mean & $S D$ & Range & Min & $\operatorname{Max}$ & Mean & $S D$ & Range & Min & $\operatorname{Max}$ \\
\hline Preoperative IOP & 14.95 & 2.91 & 10.00 & 10.50 & 20.50 & 15.82 & 2.77 & 11.00 & 10.00 & 21.00 \\
\hline \multicolumn{11}{|l|}{ Postoperative IOP } \\
\hline Day 1 & 21.12 & 2.56 & 8.50 & 16.50 & 25.00 & 19.63 & 2.75 & 11.50 & 14.00 & 25.50 \\
\hline Day 2 & 20.98 & 2.56 & 9.50 & 16.00 & 25.50 & 19.33 & 2.66 & 12.00 & 13.00 & 25.00 \\
\hline Day 7 & 18.75 & 2.91 & 10.50 & 12.50 & 23.00 & 16.95 & 2.71 & 13.00 & 10.50 & 23.50 \\
\hline Month 1 & 17.6 & 3.03 & 10.00 & 12.50 & 22.50 & 15.51 & 2.68 & 11.50 & 10.00 & 21.50 \\
\hline Month 3 & 16.96 & 3.17 & 10.50 & 12.00 & 22.50 & 13.78 & 2.79 & 12.50 & 8.00 & 20.50 \\
\hline Month 6 & 16.92 & 3.16 & 10.50 & 12.00 & 22.50 & 13.65 & 2.72 & 12.50 & 8.00 & 20.50 \\
\hline
\end{tabular}

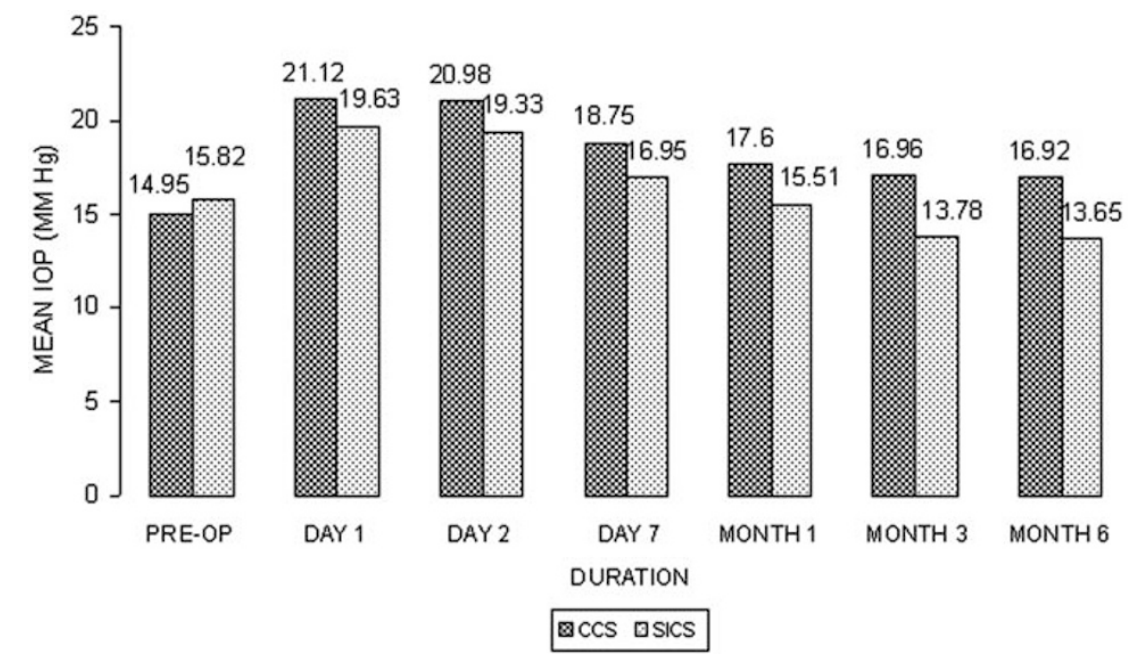

Figure 1 Distribution of mean IOP in Pre and post-op phases in CECS versus SICS

the state or charitable organizations. In this scenario, sutureless small incision cataract surgery has become very popular for its advantages over conventional surgery and therefore more and more ophthalmologists are now adopting this technique. Hence, a long-term comparative analysis of postoperative intraocular pressure in SICS merits attention, especially to prognosticate in patients of glaucoma or ocular hypertension.

In CECS, we found the mean postoperative IOP on day 1 rose to $21.12 \mathrm{~mm} \mathrm{Hg}(\mathrm{SD} \pm 2.56)$ from mean preoperative (baseline) IOP of $14.95 \pm 2.91 \mathrm{~mm} \mathrm{Hg}$, and then showed declining trend from the second postoperative day onwards till 3 months (Table 2). On the second day, mean IOP was marginally lower ( $20.98 \pm 2.56)$, but on the seventh day, it had declined substantially to $18.75 \pm 2.91 \mathrm{~mm} \mathrm{Hg}$. Thereafter, the declining trend showed a slowdown. In the next 3 weeks, mean IOP decreased by a little over $1.0 \mathrm{~mm} \mathrm{Hg}$ and in the next 2 months by less than $1.0 \mathrm{~mm} \mathrm{Hg}$. From 3 to 6 months, IOP remained almost static, thereby suggesting that IOP ceased to decline further (Figure 1). However all IOP values on all specified days were significantly higher than baseline IOP (repeated measures ANOVA, $\mathrm{F}=524.931, P<0.001)$. Multiple comparisons of rise in IOP at all specified intervals to preoperative IOP were also significant (Dunnett's test, $P<0.05$ ).

In SICS, postoperative IOP elevation followed same pattern as in CECS - a high peak $(19.63 \pm 2.75 \mathrm{~mm} \mathrm{Hg})$ from baseline $(15.82 \pm 2.77 \mathrm{~mm} \mathrm{Hg})$ on day 1 followed by mild decrease on day $2(19.33 \pm 2.66 \mathrm{~mm} \mathrm{Hg})$ and considerable decrease $(16.95 \pm 2.71 \mathrm{~mm} \mathrm{Hg})$ at 1 week. Thereafter, IOP continued to decline similarly till 3 months and remained static thereafter till the follow-up period of 6 months (Table 2; Figure 1). Repeated measures ANOVA ( $\mathrm{F}=1906.012)$ was highly significant $(P<0.001)$ and multiple comparisons to baseline IOP were also statistically significant (Dunnett's test, $P<0.05)$. 


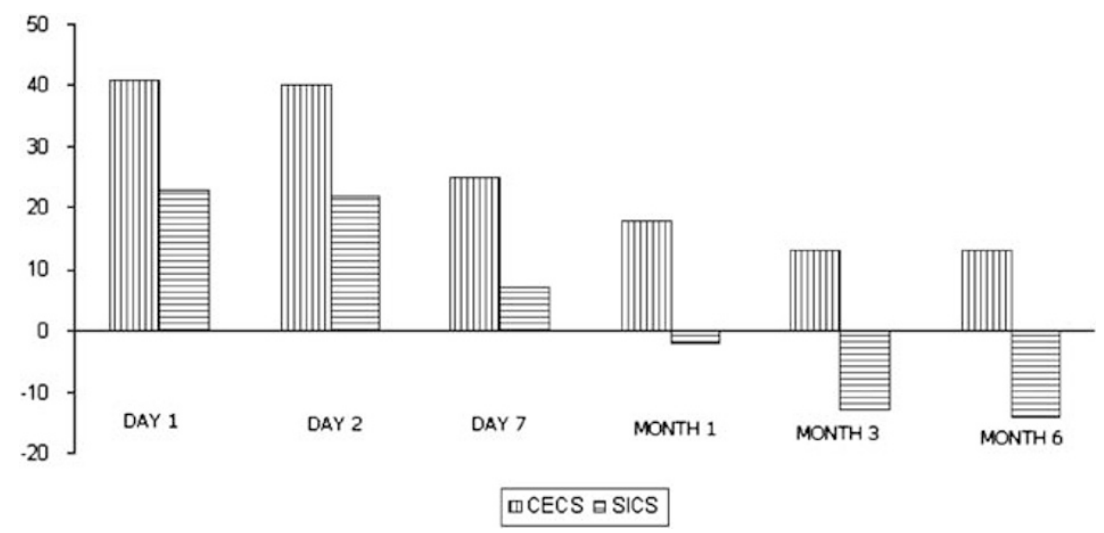

Figure 2 Chart showing distribution of percentage rise of mean IOP from preoperative level during postoperative phase.

We also compared the corresponding values of IOP rise at the specified time intervals in the two types of surgeries. As the mean preoperative IOP in the two groups were not similar, the absolute postoperative IOP values cannot be compared with each other. Therefore, the IOP was assessed in terms of percentage rise from mean preoperative IOP, which gives a more meaningful value for comparison (Figure 2). Thus on postoperative day 1 , percentage IOP rise was nearly twofold higher in CECS than in SICS. On postoperative day 2, the decrease was marginal, but by end of 1 week, it had lost 38 and $71 \%$ of the peak value in CECS and SICS respectively. At 1 month, IOP persisted $18 \%$ higher from base line in CECS, but declined below the baseline in SICS.

Thereafter, further decrease in IOP till 3 months was marginal (5\%) in CECS but significant in SICS (11\%). After this period, IOP stabilized at that level in both types of surgeries till the follow-up period of 6 months.

These observations establish the fact that in SICS, postoperative IOP rise is much less pronounced and decline is more rapid than in CECS. Maximum decline in mean IOP occurs by 1 week. At 1 month, mean IOP in SICS is marginally below the baseline IOP but much higher in CECS. At 3 and 6 months, mean IOP is below the baseline in SICS but higher than that in CECS. These observations suggest that SICS is much more beneficial and safer than CECS in glaucoma or glaucoma suspects undergoing cataract surgery.

Many authors have found similar rise in postoperative IOP in conventional cataract surgery with sutures. Gupta et $a l^{5}$ reported that IOP at $24 \mathrm{~h}$ (mean $20.3 \mathrm{~mm} \mathrm{Hg}$ ) and $48 \mathrm{~h}$ (mean $22.3 \mathrm{~mm} \mathrm{Hg}$ ) after ECCE was significantly higher than the preoperative IOP (mean $16.1 \mathrm{~mm} \mathrm{Hg}$ ) and it could effectively be controlled with intracameral carbachol during surgery. We also observed highly significant rise in IOP on these days, though the mean IOP at $48 \mathrm{~h}$ was lower than that at $24 \mathrm{~h}$ in our cases of CECS.
Wolf-Dietrich A et $a l^{7}$ found that significant IOP peaks of $30 \mathrm{~mm} \mathrm{Hg}$ or more occurred more often in cataract surgery with sclerocorneal sutures than in phacoemulsification with sclerocorneal sutures or phacoemulsification with sutureless scleral tunnel incision groups $5-7 \mathrm{~h}$ after surgery. Our results on the first postoperative day also show marked rise in IOP, though we did not record IOP at 5-7 h nor did we observe any peaks of $30 \mathrm{~mm} \mathrm{Hg}$ or more at any time.

Das et $a l^{13}$ have followed IOP changes in small incision cataract surgery over a period of 3 months. They also found mild rise in IOP in immediate postoperative period in small tunnel incision cataract surgery with a cross-stitch, but not in those who had sutureless surgery - there was instead a fall in IOP. Thus our results are at variance with these authors. They have not studied IOP in conventional extracapsular cataract surgery. Some variation in wound construction could be responsible for their observation of fall in IOP postoperatively in their small incision sutureless cataract surgery cases. However these authors have not studied the long-term effects on IOP for comparison.

Many explanations have been offered for the increased IOP seen in early postoperative period after cataract surgery. Blockage of trabecular meshwork by retained lens cortical material, retained viscoelastic, entrapped exudated serum component, pigment debris from iris, and red blood and inflammatory cells have all been blamed for this rise of IOP. ${ }^{8,14-16}$ As the surgical inflammation subsides and the residual viscoelastic and pigment debris is removed from the eye, the IOP tends to come down gradually.

The surgery itself may cause alterations in aqueous outflow by its effect on trabecular meshwork or uveoscleral pathways as well as possible alteration of blood - aqueous barrier as shown by Handa et al. ${ }^{15}$ It has been observed by Miyake et al ${ }^{17}$ that blood-ocular barrier permeability is increased after cataract surgery and IOL implantation resulting in final lowering of IOP. 
Application of corneoscleral sutures causes a watertight wound closure, which contributes to more elevation of IOP. ${ }^{6,8}$ In SICS, tunnel preparation is less traumatizing to trabeculum than a corneoscleral incision with subsequent suturing, which leads to more surgical inflammation that results in narrowing of filtration channels and reduced aqueous outflow. ${ }^{8}$ Release of prostaglandins during intraocular surgery also causes disruption of blood aqueous barrier and subsequent rise in IOP. ${ }^{18}$ Lundgren $e t ~ a l^{16}$ have shown experimentally in rabbit eyes that amount of white blood cells and prostaglandins E 2 in the anterior chamber is much higher in ECCE than in phacoemulsification.

The sutureless scleral tunnel in SICS may cause a micro leak of aqueous or its filtration through scleral bed, so as to lead to less pronounced postoperative increase in IOP, its quick reversal, and IOP lowering effect even below the baseline subsequently. Calissendroff $e t a l^{8}$ are of the opinion that there could be a leakage through the tunnel, which is not visible clinically, leading to lower postoperative IOP in small incision cataract surgery than in conventional type.

To the best of our knowledge, there is no such study in literature as this to compare our results with. Few IOP studies of scleral tunnel incision for phacoemulsification ${ }^{11-14}$ cannot be compared with this study for comparison as the incision length in this surgery is much smaller than that in SICS.

\section{Summary}

The postoperative IOP rose significantly after CECS or SICS on day 1 irrespective of the type of surgery and decreased slightly by day 2 , after which it decreased gradually to a level little above the preoperative IOP by day 7. There was further gradual decrease in IOP by months 1, 3, and 6 in both types of surgery. On all days, immediate postoperative IOP rise was less marked in SICS than in CECS. At 6 months, the IOP persisted at a little higher level than preoperative IOP in CECS and dropped below that in SICS but variations were not statistically significant. However compared with each other, IOP was significantly higher in CECS than in SICS. The study suggests that SICS leads to less pronounced IOP elevation in short term and more IOP lowering in long term in comparison to CECS. This effect may prove beneficial in the management of glaucoma patients undergoing cataract surgery in developing countries.

\section{Acknowledgements}

The authors certify that all applicable institutional and governmental regulations concerning the ethical use of human volunteers were followed during this research.

\section{References}

1 Blumenthal M. Manual ECCE, the present state of the art. Klin Monatsbl Augenheilkd 1994; 205(5): 266-270.

2 Blumenthal M, Glovinsky Y. Surgical consequences in coexisting cataract and glaucoma. Curr Opin Ophthalmol 1995; 6(2): 15-18.

3 Wedrich A, Menapace R, Stifter S. The influence of the incision length on the early postoperative intraocular pressure following cataract surgery. Int Ophthalmol 1994; 18: 77-81.

4 Haiman MH, Phelps CD. Prophylactic timolol for the prevention of high intraocular pressure after cataracat extraction. Ophthalmology 1981; 88: 233.

5 Gupta A, Bansal RK, Grewal SPS. Natural course of intraocular pressure after cataract extraction and the effect of intracameral carbachol. J Cataract Refract Surg 1992; 18: 166-169.

6 Rich WJ, Radtke ND, Cohen BE. Early ocular hypertension after cataract extraction. Br J Ophthalmol 1974; 58: 725-731.

7 Lagreze WD, Bomer TG, Funk J. Effect of surgical Technique on the increase in intraocular pressure after cataract extraction. Ophthalmic Surg Lasers 1996; 27: 169-173.

8 Calissendroff BM, Hamberg-Nystorm H. Intraocular pressure after extracapsular cataract extraction with implantation of posterior chamber lenses. Acta Ophthalmol (Copenh) 1993; 71(3): 377.

9 Gross JG, Meyer DR, Robin AL, Filar AA, Kelley JS. Increased intraocular pressure in the immediate postoperative period after extracapsular cataract extraction. Am J Ophthalmol 1988; 105: 466-469.

10 Tennen MA, Masket S. Short- and long-term effect of clear corneal incisions on intraocular pressure. J Cataract Refract Surg 1996; 22: 568-570.

11 Shingleton BJ, Gamell LS, O'Donoghue MW. Long-term changes in intraocular pressure after clear corneal phacoemulsification: normal patients versus glaucoma suspect and glaucoma patients. J Cataract Refract Surg 1999; 25: 885-890.

12 Oshima Y, Tsujikawa K, Oh A, Harino S. Comparative study of intraocular lens implantation through $3.0 \mathrm{~mm}$ temporal clear corneal and superior scleral tunnel self-sealing incisions. J Cataract Refract Surg 1997; 23: 347-353.

13 Das H, Bandhu BP, Panda A. Pattern of intraocular pressure changes following manual small incision cataract surgery. Kathmandu Univ Med I 2005; 3(4): 340-344.

14 Jürgens I, Matheu A, Castilla M. Occular hypertension after cataract surgery: a comparison of three surgical techniques and two viscoelastics. Ophthalmic Surg Lasers 1997; 28: 30-36.

15 Handa J, Henry JC, Krupin T, Keates E. Extracapsular cataract extraction with posterior chamber lens implantation in patients with glaucoma. Arch Ophthalmol 1987; 105: 765-769.

16 Lundgren B, Holst A, Torngren L, Wickstrom K. Inflammatory response after conventional extracapsular lens extraction or phacoemulsification in rabbit eyes. Invest Ophthalmol Vis Sci 1994; 35(ARVO suppl): 918.

17 Miyake K, Asakura M, Kobayashi H. Effect of intraocular lens fixation on the blood-aqueous barrier. Am J Ophthalmol 1984; 98: 451-455.

18 Sears ML. Aphakic cystoid macular edema: the pharmacology of ocular trauma. Surv Ophthalmol 1984; 28(suppl): 525-534. 